\title{
Gamma-rays from Nearby Clusters: Constraints on Selected Decaying Dark Matter Models
}

\author{
Jiwei Ke, ${ }^{*}$ Mingxing Luo, ${ }^{\dagger}$ Liucheng Wang, ${ }^{\ddagger}$ and Guohuai $\mathrm{Zhu}^{\S}$ \\ Zhejiang Institute of Modern Physics, \\ Department of Physics, Zhejiang University, \\ Hangzhou, Zhejiang 310027, P.R.China
}

(Dated: October 30, 2018)

\begin{abstract}
Recently, the Fermi-LAT collaboration reported upper limits on the $\mathrm{GeV}$ gamma-ray flux from nearby clusters of galaxies. Motivated by these limits, we study corresponding constraints on gamma-ray emissions from two specific decaying dark matter models, one via grand unification scale suppressed operators and the other via R-parity violating operators. Both can account for the PAMELA and Fermi-LAT excesses of $e^{ \pm}$. For GUT decaying dark matter, the gamma-rays from the M49 and Fornax clusters, with energy in the range of 1 to $10 \mathrm{GeV}$, lead to the most stringent constraints to date. As a result, this dark matter is disfavored with conventional model of $e^{ \pm}$background. In addition, it is likely that some tension exists between the Fermi-LAT $e^{ \pm}$ excess and the gamma-ray constraints for any decaying dark matter model, provided conventional model of $e^{ \pm}$background is adopted. Nevertheless, the GUT decaying dark matter can still solely account for the PAMELA positron fraction excess without violating the gamma-ray constraints. For the gravitino dark matter model with R-parity violation, cluster observations do not give tight constraints. This is because a different $e^{ \pm}$background has been adopted which leads to relatively light dark matter mass around $200 \mathrm{GeV}$.
\end{abstract}

\footnotetext{
* kejiwei1985@gmail.com

$\dagger$ luo@zimp.zju.edu.cn

$\ddagger$ liuchengwang@zimp.zju.edu.cn

$\S$ zhugh@zju.edu.cn;(Corresponding author)
} 


\section{INTRODUCTION}

It is now believed that the dominant matter in the universe should be non-baryonic dark matter (DM) instead of visible ones. In addition, DM should not be composed of any known Standard Model (SM) particles. There are three experimental avenues to prove the existence of DM: direct detection, indirect detection and collider searches. In this paper, we will focus on the indirect detection of DM through gamma-rays and electrons/positrons in cosmic-rays. The Pamela collaboration has reported significant excess in the positron fraction between 1 and $100 \mathrm{GeV}$ [1]. Later on the Fermi-LAT collaboration also observed a clear feature in the spectrum of electrons and positrons from $20 \mathrm{GeV}$ to $1 \mathrm{TeV}$ [2], which is harder than predictions of conventional models of cosmic ray propagation. These excesses of electrons and positrons could be attributed by the annihilation or decay of DM. If so, one might also detect gamma-rays from these DM annihilation/decay.

Recently, the Fermi-LAT collaboration has reported measurements of the GeV gamma-ray from nearby clusters of galaxies [3]. In an observation period of 18-month, there was no direct observational evidence. The upper limits on gamma-ray flux are given with $95 \%$ confidencelevel. As clusters of galaxies are DM dominated and DM annihilation/decay would almost inevitably emit gamma-rays, these upper limits may lead to stringent constraints on DM model parameters. Notice that based on observations of the first 11-month of Fermi-LAT, there already existed some discussions about DM models along this way [4-6].

To interpret the PAMELA and Fermi-LAT excesses in terms of DM annihilation, a large boost factor of order $100-1000$ is required for the theory to be consistent with the relic abundance measured by the WMAP [7-9]. Moreover, gamma-ray fluxes produced by the DM annihilation are anticipated to be large at locations with very high density of DM, because they are proportional to the local DM density squared. There are plenty of experimental measurements of gamma-rays from inside/outside the Galactic halo. Accordingly, the annihilating DM scenarios have been strongly constrained by the observations [4, 5, 10-13]. So it is not easy to build models of annihilating $\mathrm{DM}$ at $\mathrm{TeV}$ scale satisfying all constraints. ${ }^{1}$

Decaying DM offers an alternative explanation to the $e^{ \pm}$excesses. In decaying DM scenarios, constraints from gamma-ray fluxes are relatively easier to be satisfied since the gamma-ray fluxes are linearly proportional to the local DM density. However to fit the

\footnotetext{
${ }^{1}$ There are certainly ways out of this problem, see for example a recent attempt in [14]
} 
PAMELA and Fermi-LAT excesses, a lifetime of the order $10^{26}$ 's for DM is needed, which is even much longer than that of the Universe. From the perspective of particle physics, there are several frameworks which can naturally provide such a long lifetime. For instance, DM may decay via operators suppressed by the grand unification theory (GUT) scale $10^{16} \mathrm{GeV}$, which makes it sufficiently long-living. DM may also decay via a very weak R-parity violation process: R-parity conservation makes the lightest supersymmetric particle (LSP) stable to be DM candidate while R-parity violating operators make DM decay sufficiently slow. There are of course other possibilities, for example DM may decay via instanton-induced operators [15]. Recently it has also been shown that such a lifetime can arise naturally from the goldstino decay [16].

For decaying DM with $\mu^{+} \mu^{-}$and $b \bar{b}$ final states, the impact of the Fermi 11-month observation of gamma-rays from nearby clusters has been discussed in [6]. It was found that these gamma-rays from clusters considerably improves previous constraints on the lifetime and mass of the DM. Especially, the Fornax cluster provides the strongest constraint to date. This motivated us to investigate similar constraints on some (arguably) theoretically bettermotivated models of decaying DM. Specifically, we will focus on two different decaying DM scenarios mentioned above: decaying via GUT scale suppressed operators or decaying via

R-parity violating operators. As shown before, each scenario could give a reasonable fit to the $e^{ \pm}$excesses. In addition, both scenarios are consistent with measurements of Galactic and extragalactic gamma-rays. We expect that the gamma-rays from nearby clusters should lead to more stringent constraints than before.

This paper is organized as follows. We discuss the gamma-ray fluxes from galaxy clusters in general in Section II. In Section III, DM gamma-ray signals from nearby clusters are discussed in a GUT framework. Section IV is devoted to the study of a decaying DM scenario with R-parity violating operators. We conclude with a summary in section V.

\section{GAMMA-RAYS FROM CLUSTERS IN DECAYING DM SCENARIOS}

Nearby clusters are expected to be very interesting targets for DM indirect detection [17]. They are supposed to be highly DM dominated and isolated at high galactic latitudes. Thus high signal-to-noise ratios are anticipated for gamma-ray observations targeting nearby clusters. Certainly there may have other sources in clusters that can emit gamma-rays, 
besides DM annihilation/decay. Nevertheless the gamma-ray observations of nearby clusters can give upper limits to certain DM model parameters. Along this way, the EGRET gammaray measurements have been used to constrain DM annihilation models in [18]. Last year Fermi-LAT reported interesting measurements of gamma-rays from galaxy clusters of a 11month observation period, which motivated new research efforts on DM annihilation [4, 5, 19] and DM decay $[6,19]$. Recently,the Fermi-LAT collaboration have updated their results based on an observation of 18-month [3], which we will investigate in the following.

In general, the gamma-ray flux coming from a galaxy cluster can be written as

$$
\Phi\left(E_{\gamma}\right)=J(\psi) \times W\left(E_{\gamma}\right),
$$

where $J(\psi)$ and $W\left(E_{\gamma}\right)$ encode the astrophysical information and particle information, respectively. Along the direction of a cluster, $J(\psi)$ is defined as

$$
J(\psi)=\int_{\triangle \Omega} d \Omega \int d l(\psi) \rho_{D M}(l) .
$$

Here the solid angle $\triangle \Omega$ corresponds to a radius of ten degrees, since the Fermi-LAT Collaboration selected all gamma-rays within a $10^{\circ}$ radius around the direction of each galaxy cluster in their sample [3]. The parameter $l(\psi)$ is integrated along a line of sight within each cluster and $\rho_{D M}(l)$ is the DM mass density. Since $J(\psi)$ depends only on the integration over $\rho_{D M}(l)$, unlike the case of annihilating DM, the gamma-ray flux here is rather insensitive to the DM density profile.

In this paper, we will consider six galaxy clusters reported by Fermi-LAT. They are the NGC4636, M49, Fornax, Centaurus, AWM7 and Coma clusters. All of them are DM dominated and have very low gamma-ray backgrounds, as they are isolated at high galactic latitudes. The upper limits of their gamma-ray fluxes may give severe constraints on decaying DM models. More information about these galaxy clusters are listed in Table I.

Since the Fermi-LAT analysis is done within a $10^{\circ}$ radius surrounding the cluster center, the corresponding spatial extent is even larger than the cluster virial radius $R_{200}$. So we integrate over the whole cluster halo in Eq (2). Moreover, the cluster radius $R_{200}$ is much smaller than its luminosity distance $D$. Approximately as a point source, we get

$$
J(\psi) \simeq \frac{1}{D^{2}} \int d V \rho_{D M}(l)=\frac{M_{200}}{D^{2}} .
$$

So for each cluster, $J(\psi)$ is approximately independent of specific DM mass density assumption $\rho_{D M}(l)$. Using data in Table I, we find that the Fornax cluster has the largest ratio 


\begin{tabular}{|c|c|c|c|c|c|c|c|}
\hline Name & $\mathrm{z}$ & R.A. & Dec. & $R_{200}$ & $M_{200}$ & $R_{500}$ & $M_{500}$ \\
\hline \hline NGC4636 & 0.0031 & $12 \mathrm{~h} 43 \mathrm{~m}$ & $2^{\circ} 41^{\prime}$ & 0.85 & 0.35 & 0.53 & 0.22 \\
\hline M49 & 0.0033 & $12 \mathrm{~h} 30 \mathrm{~m}$ & $8^{\circ} 00^{\prime}$ & 1.04 & 0.65 & 0.66 & 0.41 \\
\hline Fornax & 0.0046 & $3 \mathrm{~h} 39 \mathrm{~m}$ & $-35^{\circ} 27^{\prime}$ & 1.35 & 1.42 & 0.84 & 0.87 \\
\hline Centaurus & 0.0114 & $12 \mathrm{~h} 49 \mathrm{~m}$ & $-41^{\circ} 18^{\prime}$ & 1.87 & 3.74 & 1.18 & 2.33 \\
\hline AWM7 & 0.0172 & $2 \mathrm{~h} 55 \mathrm{~m}$ & $41^{\circ} 35^{\prime}$ & 2.19 & 6.08 & 1.38 & 3.79 \\
\hline Coma & 0.0231 & $13 \mathrm{~h} 00 \mathrm{~m}$ & $27^{\circ} 59^{\prime}$ & 3.22 & 19.38 & 2.03 & 11.99 \\
\hline
\end{tabular}

Table I. Cluster Information. Redshifts and equatorial coordinates of clusters can be found in the NASA/IPAC Extragalactic Database. Virial radius $R_{200}$ (a radius within which the mean cluster density is 200 times larger than the critical density $\left.\rho_{c}\right), R_{500}$, and corresponding viral masses $M_{200}$, $M_{500}$ are adopted from [20]. $R_{200}$ and $R_{500}$ are in the unit of $(2 h)^{-1} \mathrm{Mpc}$, where $\mathrm{h}$ is the present day normalized Hubble expansion rate. $M_{200}$ and $M_{500}$ are in the unit of $(2 h)^{-1} 10^{14} M_{\odot}$, where $M_{\odot}$ is the solar mass.

$M_{200} / D^{2}$, which should have the brightest gamma-ray emission from decaying DM.

Now we turn to the particle physics factor $W\left(E_{\gamma}\right)$, which contains the mechanism to produce photons in decaying DM scenarios. The gamma-ray flux comes from final state radiations (FSR) and the inverse Compton scattering (ICS).

(1) FSR: Inevitably the bremsstrahlung of $e^{ \pm}$interacting with interstellar and/or intracluster gas leads to the emission of energetic photon. In addition, if $\tau$ lepton, for example, exists in the decay process, $\tau \rightarrow \pi^{0} \rightarrow \gamma+\gamma$ would also produce gamma-rays. FSR is quite model-dependent and all decay channels involving photons should be taken into account. Notice that photons propagate almost freely, the FSR flux of photons from a cluster is given by

$$
\Phi\left(E_{\gamma}\right)=\frac{M_{200}}{4 \pi D^{2}} \sum_{i} \frac{\Gamma_{i}^{D M}}{M^{D M}} \frac{d N_{i}^{D M}}{d E_{\gamma}} .
$$

Here the summation is over all possible decay channels which may produce gamma-rays. $\Gamma_{i}^{D M}$ is the decay width of a specific channel and $M^{D M}$ is the DM mass. $d N_{i}^{D M} / d E_{\gamma}$ is the photon spectrum per DM decay via a specific channel. PYTHIA package [21] has been used in our calculation to obtain these spectra numerically.

(2) ICS: The ICS radiation is produced when the energetic $e^{ \pm}$scatter on the interstellar radiation field (ISRF) in galaxy clusters. A pedagogical review of ICS $W\left(E_{\gamma}\right)$ is provided 
in [22]. We calculate it semi-analytically, following Refs. [23-28]. Noticing that the CMB component is dominant in the ISRF in each cluster. Combining $J(\psi)$ and $W\left(E_{\gamma}\right)$, we obtain the ICS flux of photons from a cluster

$$
\Phi\left(E_{\gamma}\right)=\frac{M_{200}}{4 \pi D^{2}} \sum_{i} \frac{\Gamma_{i}^{D M}}{M^{D M}} \int d \epsilon f_{C M B}(\epsilon) \int d E_{e} \frac{d \sigma^{I C S}\left(E_{e}, \epsilon\right)}{d E_{\gamma}} \frac{d N_{i}^{D M}\left(E_{e}\right)}{d E_{e}} .
$$

Here the summation is over all possible decay channels with electron and/or positron in the final states. The number density of CMB background $f_{C M B}(\epsilon)$ should be a blackbody-like spectrum [23]

$$
f_{C M B}(\epsilon)=\frac{\epsilon^{2}}{\pi^{2}} \frac{1}{e^{\epsilon / T}-1}
$$

with $T=2.753 \mathrm{~K}$. The initial electron/positron spectrum may be solved from the diffusionloss equation. Approximately,

$$
\frac{d N_{i}^{D M}\left(E_{e}\right)}{d E_{e}}=\frac{1}{B\left(E_{e}\right)} \int_{E_{e}}^{M_{D M}} d E^{\prime} \frac{d N_{i}^{D M}}{d E^{\prime}}
$$

where $B\left(E_{e}\right)=E^{2} /\left(G e V \cdot \tau_{E}\right)$ is the effective energy loss coefficient with $\tau_{E}=4 \times 10^{16} \mathrm{~s}$. The Compton cross section is given by the Klein-Nishina formula

$$
\frac{d \sigma^{I C S}\left(E_{e}, \epsilon\right)}{d E_{\gamma}}=\frac{3 \sigma_{T}}{4 \gamma_{e}^{2} \epsilon}\left(2 q \ln q+1+q-2 q^{2}+\frac{(q \Gamma)^{2}}{2(1+q \Gamma)}(1-q)\right)
$$

where

$$
q=\frac{E_{\gamma}}{\Gamma\left(E_{e}-E_{\gamma}\right)}, \Gamma=\frac{4 \gamma_{e} \epsilon}{m_{e}}, \gamma_{e}=\frac{E_{e}}{m_{e}} .
$$

$m_{e}$ denotes the mass of electron and $\sigma_{T}=0.67$ barn is the Compton scattering cross section in the Thomson limit. For energy integration, $\epsilon \leq E_{\gamma} \leq\left[\left(1 / E_{e}+1 /\left(4 \gamma_{e}^{2} \epsilon\right)\right]^{-1}\right.$ is required by kinematics.

\section{DECAYING DARK MATTER IN GRAND UNIFICATION THEORY}

The possibility that the Pamela positron fraction excess may be accounted for by the GUT suppressed operators was first discussed in [29]. Since then, many decaying DM models in the framework of GUT have been proposed [27, 30-37]. To quantitatively study the possible gamma-ray signals from clusters, we choose one of the DM models as a template [27]. First

we briefly review the main features of this model. An SU(5) singlet $S$ is introduced as the 

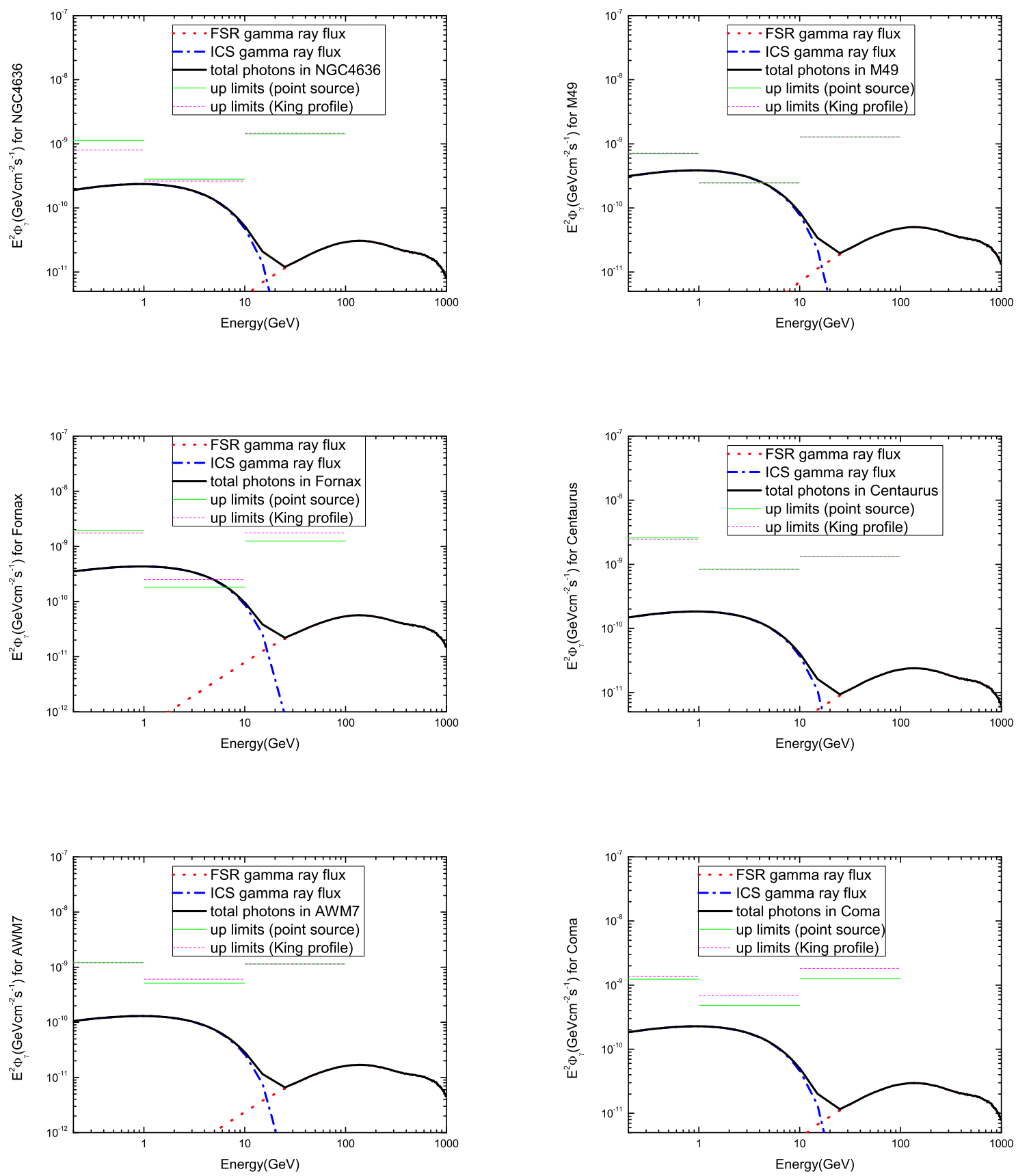

Figure 1. In a GUT DM model, gamma-ray spectra are shown for NGC4636 (top left), M49 (top right), Fornax (middle left), Centaurus (middle right), AWM7 (bottom left) and Coma (bottom right) clusters. Experimental upper limits are from the Fermi-LAT collaboration [3].

DM candidate. For $S$ to be stable, a discrete $Z_{2}$ symmetry is assumed. $S$ couples to the MSSM particles via dimension six operators suppressed by GUT scale $M_{G U T} \sim 10^{16} \mathrm{GeV}$

$$
\frac{S^{+} S \overline{5}^{+} \overline{5}}{M_{G U T}^{2}}, \quad \frac{S^{+} S T r\left(10^{+} 10\right)}{M_{G U T}^{2}} .
$$


Here $S=\tilde{s}+\sqrt{2} \theta s+\theta^{2} F_{s}$ is a standard chiral superfield. $\overline{5}$ is the anti-fundamental representation of $\mathrm{SU}(5)$ and 10 is the antisymmetric tensor representation.

For DM decay, we further assume the $Z_{2}$ symmetry to be spontaneously broken. The scalar field of $S$ develops a vacuum expectation value (VEV), making both fields $(\widetilde{s}, s)$ in $S$ decay via the operators of Eq.(10). In addition, the squark masses are assumed to be so heavy that DM decays dominantly into sleptons. Assuming the DM is mainly composed of scalar $\tilde{s}$, the operators of Eq.(10) can be expanded in terms of component fields as

$$
\sum_{\widetilde{l}} \frac{-1}{M_{G U T}^{2}}<\widetilde{s}>\widetilde{s}^{*}\left({\widetilde{l_{L}}}^{*} \square \widetilde{l_{L}}+\widetilde{l_{R}} \square \widetilde{l_{R}}{ }^{*}\right) .
$$

Here $\widetilde{l}$ denotes the sleptons $\widetilde{e}, \widetilde{\mu}$ and $\widetilde{\tau}$. With R-parity conservation, the slepton would decay to the LSP and lepton subsequently. $e^{ \pm}$and $\gamma$ can be produced through the following cascade decay chains: (1) selectron chain $\widetilde{s} \rightarrow \widetilde{e} \rightarrow e / \gamma ;(2)$ smuon chain $\widetilde{s} \rightarrow \widetilde{\mu} \rightarrow \mu \rightarrow e / \gamma$ and (3) stau chain $\widetilde{s} \rightarrow \widetilde{\tau} \rightarrow \tau \rightarrow e / \gamma$. Numerically, PYTHIA package [21] is used to get the spectra of $e^{ \pm}$and $\gamma$.

For interstellar $e^{ \pm}$background fluxes, we adopt "model 0" presented by the Fermi-LAT [2]. Reasonable fits to PAMELA $e^{+} /\left(e^{+}+e^{-}\right)$fraction and Fermi-LAT $e^{+}+e^{-}$spectrum can be obtained [27] with the illustrative parameter set: DM mass $M_{\widetilde{s}}=6.5 \mathrm{TeV}, M_{G U T}=10^{16}$ $\mathrm{GeV}$, the VEV $<\widetilde{s}>=20 \mathrm{TeV}$, selectron mass $M_{\widetilde{e}}=380 \mathrm{GeV}$, smuon mass $M_{\tilde{\mu}}=370 \mathrm{GeV}$, stau mass $M_{\widetilde{\tau}}=330 \mathrm{GeV}$ and LSP particle mass $M_{L S P}=300 \mathrm{GeV}$. Moreover, gamma-rays emitted from DM decay are consistent with the Fermi-LAT data in the region $0^{\circ} \leq l \leq$ $360^{\circ}, 10^{\circ} \leq|b| \leq 20^{\circ}$.

With the same parameter set, the gamma-ray signals coming from six nearby clusters are compared with the upper limits measured recently by the Fermi-LAT collaboration [3], as shown in Fig. 1. The expected signals and experimental upper limits are comparable in magnitude for all six clusters. Therefore clusters are good targets to constrain or even falsify DM models. As we can see, this GUT DM model already predicts too much gamma-rays from the M49 and Fornax clusters in the energy range 1-10 GeV, which are in disagreement with the Fermi-LAT observations. Coincidentally, the $1-10 \mathrm{GeV}$ energy band is also the most sensitive range of the Fermi-LAT detector. In this energy range the gamma rays come mainly from the ICS process, as can be seen from Fig. 1. As the CMB photons are well measured, ICS process is largely determined by the spectra of initial electrons and positrons. Notice that for all decaying DM models which could account for the PAMELA and Fermi- 

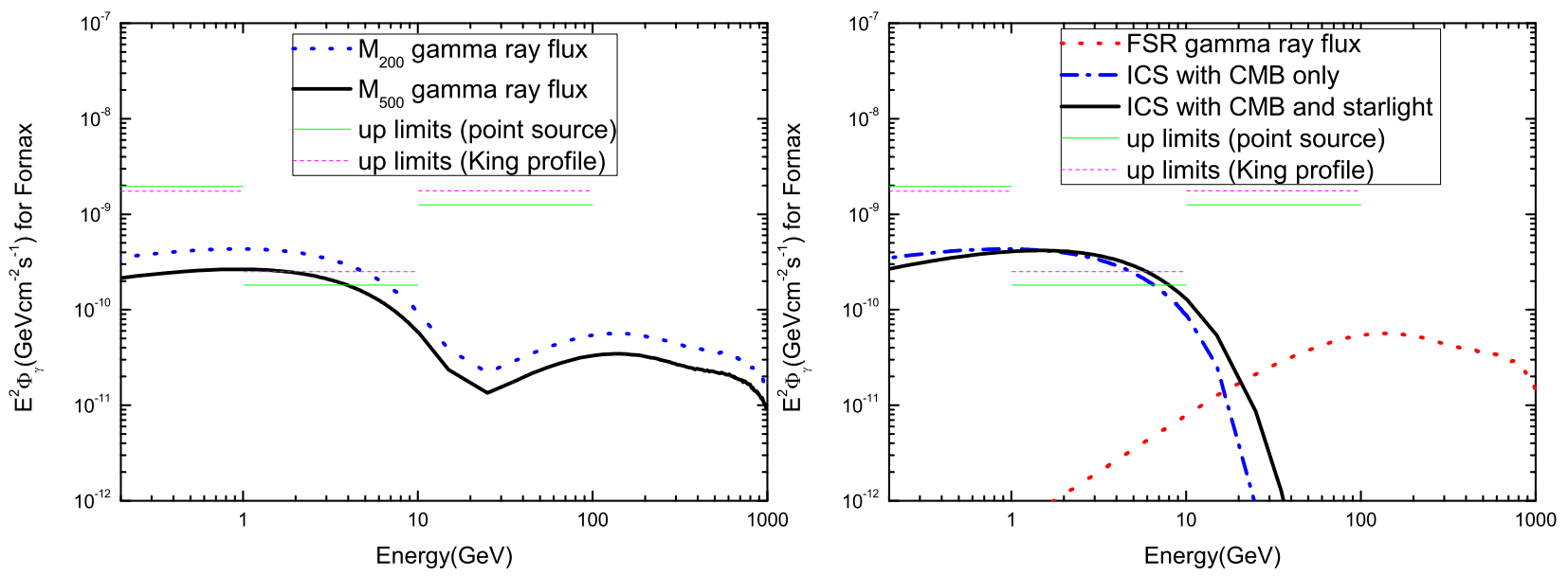

Figure 2. Left: the uncertainty of total DM mass in Fornax cluster, the thick solid curve represents the gamma-ray flux with virial mass $M_{500}$. Right: estimating the starlight in the Fornax cluster in a naive way. The corresponding ICS flux of photons is shown as a thick solid curve. To contrast, the standard gamma-ray fluxes are reproduced from Fig 1.

LAT $e^{ \pm}$excesses, the produced spectra of electrons and positrons should be more or less the same provided the same $e^{ \pm}$background is used. In this sense, the ICS flux of photons is model-independent to a large extent. So it is likely that, for any decaying dark matter model, some tension exists between the Fermi-LAT $e^{ \pm}$excess and the gamma-ray constraints from nearby clusters if conventional model of $e^{ \pm}$background is adopted.

We now address the theoretical uncertainties of gamma-ray signals. As we have taken the point source approximation in Section II, the Fornax cluster gives a severer constraint than the M49 cluster does. So let's take the Fornax cluster as an example. One source of uncertainties is related to the total DM mass in the Fornax cluster. For example, if we choose virial radius $R_{500}$ instead of $R_{200}$, the correspondingly total DM mass is $M_{500}$, which is less than $M_{200}$. Even so our model still overproduces gamma-ray flux, as shown on the left of Fig 2. Another source of uncertainties is that only the CMB photons are considered in the ICS process. In principle, the energetic $e^{ \pm}$may also scatter on the starlight in the Fornax cluster, which may lead to a harder gamma-ray spectrum. However, it is rather difficult to quantitatively estimate the effect of starlight in the Fornax cluster, though qualitatively one might expect that this effect should not be too large for such a DM dominant system. In order to have a feeling on the potential starlight effect, we relate naively the starlight of 

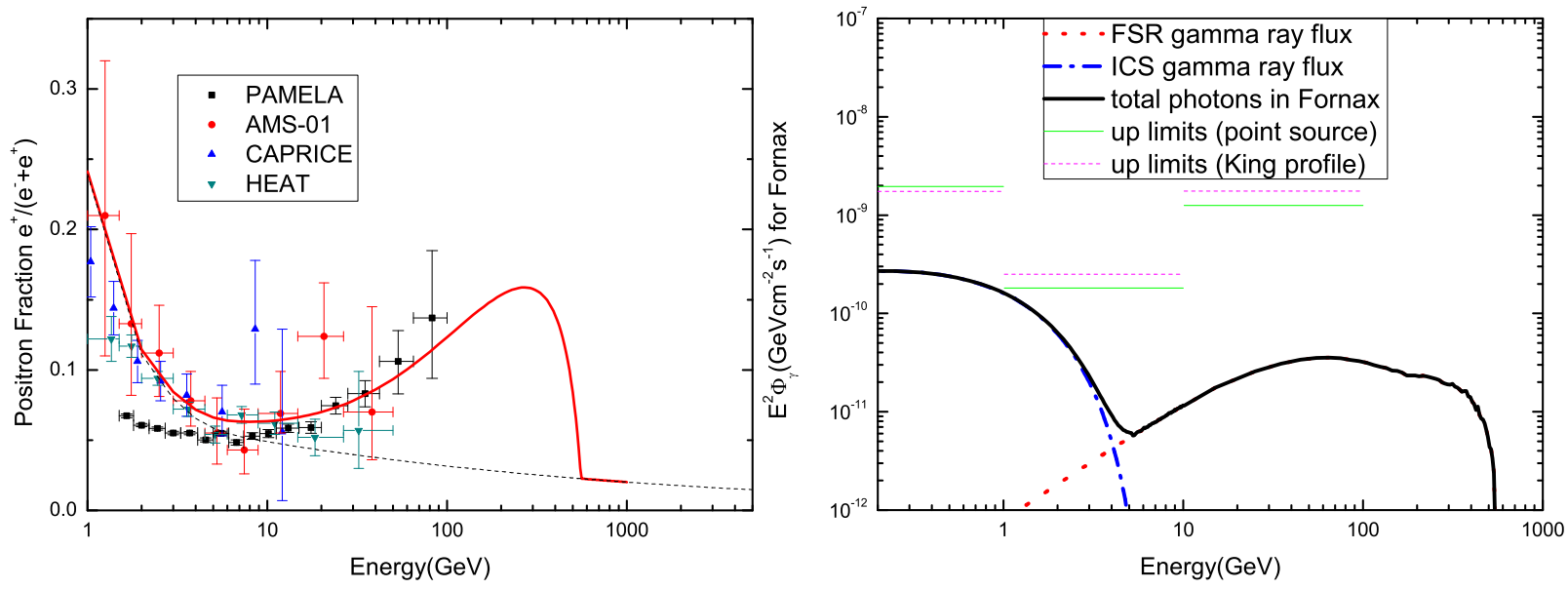

Figure 3. New parameter set in a GUT decaying DM model. Left: the predicted positron fraction is consistent with experimental data. The solid line shows the fit and the dash line denotes the background. Right: DM induced gamma-ray fluxes in the Fornax cluster, which are below the Fermi-LAT upper limits.

Fornax cluster to that of our Milky Way by a dimensional analysis

$$
\frac{f_{\text {Fornax }}}{f_{\text {Milky }}}=\frac{L_{\text {Fornax }} / R_{\text {Fornax }}^{3}}{L_{\text {Milky }} / R_{\text {Milky }}^{3}},
$$

where $f$ is the starlight photon density. The total luminosity $L$ in the Milky Way is about $10^{44} \mathrm{erg} / \mathrm{s}$ while the Fornax luminosity is taken from [20]. Choosing the density $f_{\text {Milky }}$ in the range $0^{\circ} \leq l \leq 360^{\circ}, 10^{\circ} \leq|b| \leq 20^{\circ}$ as a typical value, the starlight density $f_{\text {Fornax }}$ can be estimated. The resulting gamma-ray spectrum is shown in the right of Fig 2, which indicates that the impact of starlight on the gamma-rays is indeed rather limited. Though the ICS spectrum does become harder as expected, it still violates the Fermi-LAT constraints in the energy range $1-10 \mathrm{GeV}$.

The way out of this problem may lie in the change of cosmic ray background. For example, if the Fermi-LAT electrons excess is due to unidentified astrophysical sources instead of DM decay, a new parameter set can be chosen to fit the Pamela excess: $M^{D M}=3 \mathrm{TeV}$, $M_{G U T}=10^{16} \mathrm{GeV},<\widetilde{s}>=11 \mathrm{TeV}, M_{\tilde{e}}=380 \mathrm{GeV}, M_{\tilde{\mu}}=370 \mathrm{GeV}, M_{\tilde{\tau}}=330 \mathrm{GeV}$ and $M_{L S P}=300 \mathrm{GeV}$. In this case the DM mass is smaller by a factor of two, correspondingly the DM induced gamma-ray spectrum becomes softer. Then the gamma-ray flux upper limits from the Fornax cluster can be satisfied, as shown in Fig. 3. It may also be possible that the Fermi-LAT electrons excess is accounted for partly by the change of electrons background 
and partly by the DM contribution. We will discuss this possibility in the next section, though in a different decaying DM model with R-parity violation.

\section{DECAYING DARK MATTER WITH R-PARITY VIOLATION}

In R-parity violating SUSY models, the gravitino is an interesting DM candidate as primordial nucleosynthesis, thermal leptogenesis and gravitino DM are naturally consistent [38]. Motivated by the PAMELA and Fermi-LAT anomalies, considerable efforts have been devoted to study decaying DM in the framework of SUSY models with R-parity violation [39-48].

Phenomenologically, the trilinear operator $L_{i} L_{j} \bar{E}_{3}$ with the gravitino mass heavier than 1.5 TeV could fit the Pamela and Fermi-LAT data [47], where $L / E$ are the left-handed lepton doublet/singlet superfields. Since both the GUT decaying DM model and the above gravitino DM model involve multibody decay processes, the produced spectra of electrons and positrons in both models should be more or less the same to explain the Pamela and Fermi-LAT excesses. But as discussed in the previous section, gamma rays in the energy range $1-10 \mathrm{GeV}$ come mainly from the ICS process which is largely determined by electrons and positrons produced from the DM decays. Therefore it is likely that, similar to the case of GUT decaying DM, the $L L \bar{E}$-type gravitino model would also predict too much gamma-rays from the Fornax clusters in the energy range $1-10 \mathrm{GeV}$ as compared with the Fermi-LAT observations.

Here, we will focus instead on a decaying gravitino $\left(\psi_{\mu}\right)$ DM model [39], where R-parity violating operators are introduced in the soft supersymmetry breaking lagrangian as

$$
\mathcal{L}_{R P V}=B_{i} \tilde{L}_{i} H_{u}+M_{\tilde{L}_{i}, H_{d}}^{2} \tilde{L}_{i} H_{d}^{*}+H . C .
$$

Here $H_{u}$ and $H_{d}$ are up-type and down-type Higgs doublet, respectively. $\tilde{L}_{i}$ is left-handed slepton doublet, the index $i=1,2,3$ denotes the sleptons $\widetilde{e}, \widetilde{\mu}$ and $\widetilde{\tau}$, respectively. R-parity and the lepton number are spontaneously breaking if the left-handed sneutrino field $\tilde{\nu}_{i}$ gets a VEV $<\tilde{\nu}_{i}>$. Then the DM particle $\psi_{\mu}$ will no longer be absolutely stable. By integrating out heavier fields, one can obtain the effective lagrangian governing the $\psi_{\mu}$ decay, as discussed in [39]. A proper small $<\tilde{\nu}_{i}>$ would lead to the lifetime of DM around $10^{26} \mathrm{~s}$. There are various cascade decay chains which may produce energetic electrons, positrons and gamma- 
rays: (1) $Z$ channel $\psi_{\mu} \rightarrow Z+\nu \rightarrow e / \gamma$; (2) $W$ channel $\psi_{\mu} \rightarrow W+l \rightarrow e / \gamma$; (3) Higgs channel $\psi_{\mu} \rightarrow h+\nu \rightarrow e / \gamma$ and (4) Monochromatic channel $\psi_{\mu} \rightarrow \gamma+\nu$ (no $e^{ \pm}$final state). The relative importance of different decay chains depends very sensitively on the DM mass. Again PYTHIA package [21] has been used in order to describe the hadronization process and get the energy spectra of $e^{ \pm}$and $\gamma$.

Phenomenology of this model has been discussed in detail in [40]. Assuming $B_{1} \gg B_{2}$, $B_{3}$, the DM $\psi_{\mu}$ will decay mainly into the first generation leptons plus $\gamma, \mathrm{W}, \mathrm{Z}$ and Higgs bosons. Assuming large Higgsino-mass limit and gaugino mass unification, the parameters can be chosen as: DM mass $M_{\psi_{\mu}}=200 \mathrm{GeV}$, DM lifetime $\tau_{\psi_{\mu}}=9.6 \times 10^{26} \mathrm{~s}$, Higgs mass $M_{h}=115 \mathrm{GeV}$, neutralino mass $M_{\tilde{\chi}}=1.5 M_{\psi_{\mu}}$ and sneutrino mass $M_{\tilde{\nu}}=2 M_{\psi_{\mu}}$. In this parameter set, the $\psi_{\mu}$ decay is dominated by the $Z$ channel and $W$ channel. Notice that the DM mass here is relatively light, only $200 \mathrm{GeV}$. This is because the decay final states of $\mathrm{W}$ and $\mathrm{Z}$ bosons contain plenty of hadrons, especially protons and antiprotons. However, no excess of antiproton was found by the PAMELA collaboration [49]. In order to avoid the overproduction of anti-proton, DM with a larger mass is disfavored in this model. Obviously, such a relatively light DM can not produce electrons and positrons with energy above $100 \mathrm{GeV}$, which falls short of explaining the Fermi-LAT $e^{ \pm}$excess. To solve this problem, different $e^{ \pm}$backgrounds have to be considered, as discussed in [40]. The primary $e^{ \pm}$are supposed to originate from supernova remnant, which obeys a power law distribution. In [40], the normalization factor and power index are chosen to be different from the conventional "model 0" background. This leads to harder but still plausible backgrounds, which can fit the Fermi-LAT $e^{+}+e^{-}$spectrum above $100 \mathrm{GeV}$. Adopting this $e^{ \pm}$backgrounds, the decaying DM model with R-parity violation could predict $e^{ \pm}$spectra agreeing with the PAMELA and Fermi-LAT observations.

With the same parameter set, the DM induced gamma-ray fluxes from nearby clusters are predicted to be consistent with the Fermi-LAT observations, as can be seen from Fig. 4. This is because the relatively light DM mass $M_{\psi_{\mu}}=200 \mathrm{GeV}$ in this scenario can only produce electrons and positrons with energy below $100 \mathrm{GeV}$. That means the ICS process hardly emit gamma-rays above $0.1 \mathrm{GeV}$ due to kinematics. Then the main contribution to $\mathrm{GeV}$ gamma-ray comes from FSR process in this model. It should be kept in mind that this interpretation relies on harder $e^{ \pm}$backgrounds. With this choice of background, one may also decrease the mass of decaying DM in the framework of GUT to satisfy the gamma-ray 
constraints from the clusters. In any case, it predicts the PAMELA positron excess to be disappear with energy above $100 \mathrm{GeV}$, which can definitely be checked in the near future.

\section{SUMMARY}

In this paper, we have studied the gamma-ray signals from six nearby clusters of galaxies in decaying DM scenarios. Specifically, we have concentrated on two decaying DM models which are physically well motivated. One is decaying DM in the framework of GUT and the other is decaying DM with R-parity violation. As discussed in previous works, both of them are able to account for the observational $e^{ \pm}$excesses and consistent with various measurements of gamma-rays from inside/outside the Galactic halo. Recently, the FermiLAT collaboration has reported new upper limits on GeV gamma-ray fluxes from clusters of galaxies. We use these limits to further constrain decaying DM scenarios.

For GUT decaying DM, we find that the DM induced gamma-ray signals are comparable in magnitude to the experimental upper limits for all six clusters. Too much gamma-ray is predicted to come from the M49 and Fornax clusters in the range $1-10 \mathrm{GeV}$, which are in disagreement with Fermi-LAT observations. This conclusion remains unchanged even if we include the uncertainties of the total DM mass and the starlight in the cluster. In this energy range most contributions come from the ICS process, which are model-independent to a large extent. With the conventional astrophysical background of electrons and positrons, it seems unlikely for any decaying DM models to account for the PAMELA and Fermi-LAT excesses under the constraints of $\mathrm{GeV}$ gamma-ray flux upper limits from nearby clusters. However, it is possible that the Fermi-LAT electrons excess is due to, for instance, unidentified astrophysical sources instead of DM decay. In this case, the GUT decaying DM could interpret the PAMELA positron fraction excess and induce gamma-ray fluxes consistent with observations from nearby clusters.

Unlike the GUT decaying DM model, the gamma-ray fluxes from nearby clusters are predicted to be consistent with Fermi-LAT observations for decaying DM scenario with Rparity violation. This is because the DM mass here is just around $200 \mathrm{GeV}$. With such a relatively light DM, the ICS process hardly emits gamma-rays above $0.1 \mathrm{GeV}$ due to kinematics. The contribution to GeV gamma-ray flux comes dominantly from the FSR

process. It should be kept in mind that in order to account for the Fermi-LAT $e^{ \pm}$excess in 
this model, astrophysical $e^{ \pm}$background harder than conventional one has to be adopted. As a result, such interpretation would require the PAMELA positron excess to disappear at energies above $100 \mathrm{GeV}$, which could be checked in the near future.

\section{ACKNOWLEDGMENTS}

We thank Xiao-Jun Bi for helps on astrophysics and Wei Wu for useful discussions on PYTHIA package. This work is supported in part by the National Science Foundation of China (No.10875103, No. 11075139 and No.10705024) and National Basic Research Program of China (2010CB833000). M.L and G.Z are also supported in part by the Fundamental Research Funds for the Central Universities.

[1] O. Adriani et al. (PAMELA), Nature, 458, 607 (2009), arXiv:0810.4995 [astro-ph].

[2] A. A. Abdo et al. (The Fermi LAT), Phys. Rev. Lett., 102, 181101 (2009), arXiv:0905.0025 [astro-ph.HE].

[3] M. Ackermann et al. (Fermi-LAT), (2010), arXiv:1006.0748 [astro-ph.HE].

[4] M. Ackermann et al., JCAP, 1005, 025 (2010), arXiv:1002.2239 [astro-ph.CO].

[5] Q. Yuan, P.-F. Yin, X.-J. Bi, X.-M. Zhang, and S.-H. Zhu, Phys. Rev., D82, 023506 (2010), arXiv:1002.0197 [astro-ph.HE].

[6] L. Dugger, T. E. Jeltema, and S. Profumo, JCAP, 1012, 015 (2010), arXiv:1009.5988 [astroph.HE].

[7] L. Bergstrom, T. Bringmann, and J. Edsjo, Phys. Rev., D78, 103520 (2008), arXiv:0808.3725 [astro-ph].

[8] I. Cholis, L. Goodenough, D. Hooper, M. Simet, and N. Weiner, Phys. Rev., D80, 123511 (2009), arXiv:0809.1683 [hep-ph].

[9] M. Cirelli, M. Kadastik, M. Raidal, and A. Strumia, Nucl. Phys., B813, 1 (2009), arXiv:0809.2409 [hep-ph].

[10] E. Nardi, F. Sannino, and A. Strumia, JCAP, 0901, 043 (2009), arXiv:0811.4153 [hep-ph].

[11] G. Bertone, M. Cirelli, A. Strumia, and M. Taoso, JCAP, 0903, 009 (2009), arXiv:0811.3744 [astro-ph]. 
[12] M. Cirelli, P. Panci, and P. D. Serpico, Nucl. Phys., B840, 284 (2010), arXiv:0912.0663 [astro-ph.CO].

[13] M. Papucci and A. Strumia, JCAP, 1003, 014 (2010), arXiv:0912.0742 [hep-ph].

[14] Z.-P. Liu, Y.-L. Wu, and Y.-F. Zhou, (2011), arXiv:1101.4148 [hep-ph].

[15] C. D. Carone, J. Erlich, and R. Primulando, Phys. Rev., D82, 055028 (2010), arXiv:1008.0642 [hep-ph].

[16] H.-C. Cheng, W.-C. Huang, I. Low, and A. Menon, (2010), arXiv:1012.5300 [hep-ph].

[17] T. E. Jeltema, J. Kehayias, and S. Profumo, Phys. Rev., D80, 023005 (2009), arXiv:0812.0597 [astro-ph].

[18] A. Pinzke, C. Pfrommer, and L. Bergstrom, Phys. Rev. Lett., 103, 181302 (2009), arXiv:0905.1948 [astro-ph.HE].

[19] A. J. Cuesta et al., (2010), arXiv:1007.3469 [astro-ph.HE].

[20] T. H. Reiprich and H. Boehringer, Astrophys. J., 567, 716 (2002), arXiv:astro-ph/0111285.

[21] T. Sjostrand, S. Mrenna, and P. Skands, JHEP, 05, 026 (2006), arXiv:hep-ph/0603175.

[22] G. R. Blumenthal and R. J. Gould, Rev. Mod. Phys., 42, 237 (1970).

[23] M. Cirelli and P. Panci, Nucl. Phys., B821, 399 (2009), arXiv:0904.3830 [astro-ph.CO].

[24] A. Ibarra, D. Tran, and C. Weniger, JCAP, 1001, 009 (2010), arXiv:0906.1571 [hep-ph].

[25] A. Ibarra, D. Tran, and C. Weniger, Phys. Rev., D81, 023529 (2010], arXiv:0909.3514 [hep$\mathrm{ph}]$.

[26] K. Ishiwata, S. Matsumoto, and T. Moroi, Phys. Lett., B679, 1 (2009), arXiv:0905.4593 [astro-ph.CO].

[27] M. Luo, L. Wang, W. Wu, and G. Zhu, Phys. Lett., B688, 216 (2010), arXiv:0911.3235 [hep-ph].

[28] J. Zhang, Q. Yuan, and X.-J. Bi, Astrophys. J., 720, 9 (2010), arXiv:0908.1236 [astro-ph.HE].

[29] C.-R. Chen, F. Takahashi, and T. T. Yanagida, Phys. Lett., B673, 255 (2009), arXiv:0811.0477 [hep-ph].

[30] A. Arvanitaki et al., Phys. Rev., D79, 105022 (2009), arXiv:0812.2075 [hep-ph].

[31] A. Arvanitaki et al., Phys. Rev., D80, 055011 (2009), arXiv:0904.2789 [hep-ph].

[32] J. T. Ruderman and T. Volansky, (2009), arXiv:0907.4373 [hep-ph].

[33] J. T. Ruderman and T. Volansky, JHEP, 02, 024 (2010), arXiv:0908.1570 [hep-ph].

[34] M. Kadastik, K. Kannike, and M. Raidal, Phys. Rev., D80, 085020 (2009), arXiv:0907.1894 
[hep-ph].

[35] B. Kyae, Phys. Lett., B685, 19 (2010), arXiv:0909.3139 [hep-ph].

[36] N. Haba, Y. Kajiyama, S. Matsumoto, H. Okada, and K. Yoshioka, Phys. Lett., B695, 476 (2011], arXiv:1008.4777 [hep-ph].

[37] X. Gao, Z. Kang, and T. Li, Eur. Phys. J., C69, 467 (2010), arXiv:1001.3278 [hep-ph].

[38] W. Buchmuller, L. Covi, K. Hamaguchi, A. Ibarra, and T. Yanagida, JHEP, 03, 037 (2007), arXiv:hep-ph/0702184.

[39] K. Ishiwata, S. Matsumoto, and T. Moroi, Phys. Rev., D78, 063505 (2008), arXiv:0805.1133 [hep-ph].

[40] K. Ishiwata, S. Matsumoto, and T. Moroi, JHEP, 12,006 (2010), arXiv:1008.3636 [hep-ph].

[41] K. Ishiwata, S. Matsumoto, and T. Moroi, JHEP, 05, 110 (2009), arXiv:0903.0242 [hep-ph].

[42] S. Shirai, F. Takahashi, and T. T. Yanagida, Phys. Lett., B680, 485 (2009), arXiv:0905.0388 [hep-ph].

[43] S.-L. Chen, R. N. Mohapatra, S. Nussinov, and Y. Zhang, Phys. Lett., B677, 311 (2009), arXiv:0903.2562 [hep-ph].

[44] K. Hamaguchi, F. Takahashi, and T. T. Yanagida, Phys. Lett., B677, 59 (2009), arXiv:0901.2168 [hep-ph].

[45] G. Vertongen and C. Weniger, (2011), arXiv:1101.2610 [hep-ph].

[46] K.-Y. Choi, D. Restrepo, C. E. Yaguna, and O. Zapata, JCAP, 1010, 033 (2010), arXiv:1007.1728 [hep-ph].

[47] N. E. Bomark, S. Lola, P. Osland, and A. R. Raklev, Phys. Lett., B686, 152 (2010), arXiv:0911.3376 [hep-ph].

[48] W. Buchmuller, A. Ibarra, T. Shindou, F. Takayama, and D. Tran, JCAP, 0909, 021 (2009), arXiv:0906.1187 [hep-ph].

[49] O. Adriani et al. (PAMELA), Phys. Rev. Lett., 105, 121101 (2010), arXiv:1007.0821 [astroph.HE]. 

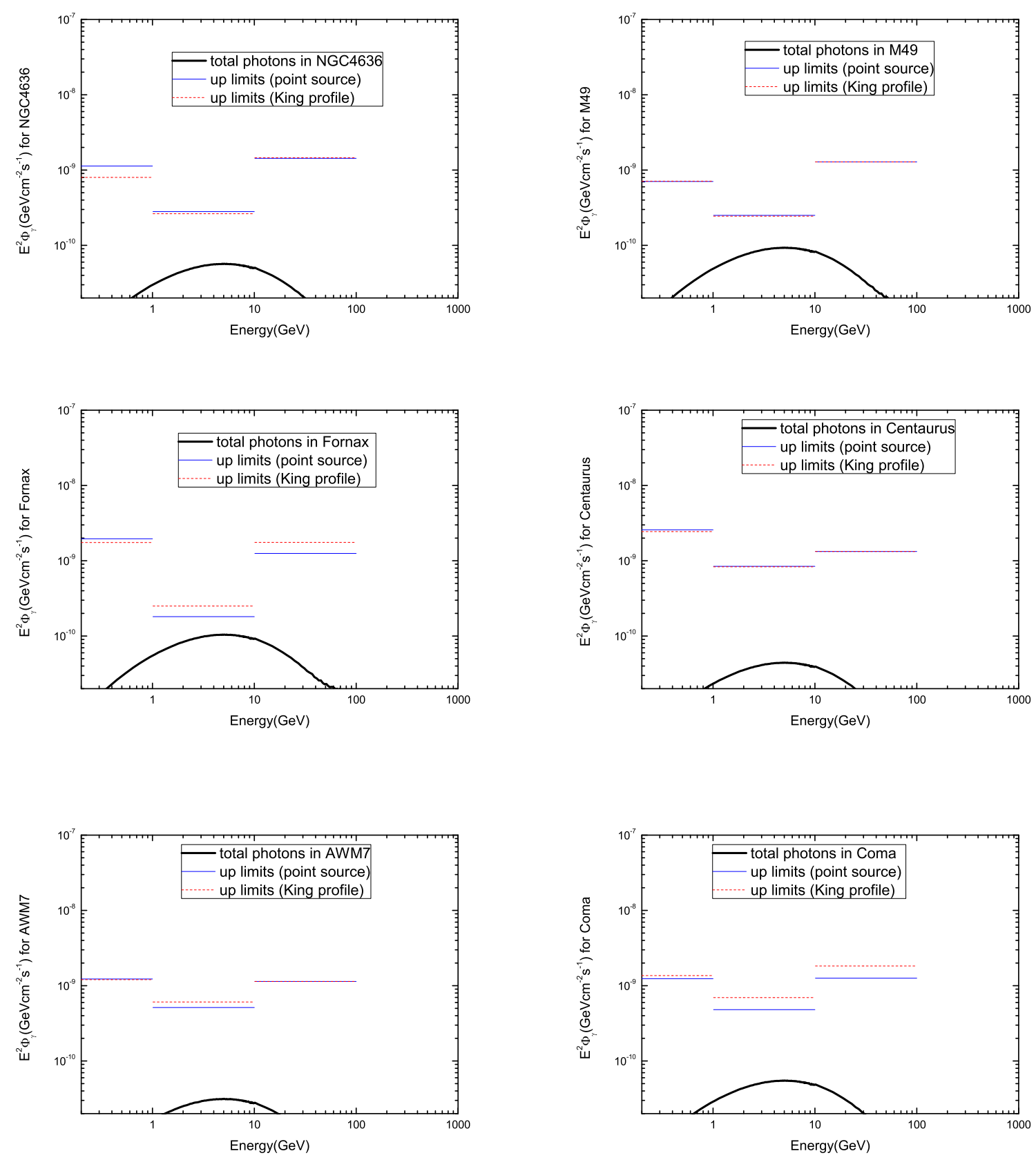

Figure 4. For decaying DM with R-parity violation, gamma-ray spectra from NGC4636 (top left), M49 (top right), Fornax (middle left), Centaurus (middle right), AWM7 (bottom left) and Coma (bottom right) clusters are shown. Experimental upper limits are from the Fermi-LAT collaboration [3]. The main contribution to GeV gamma-ray signals comes from FSR process in this model 